\title{
Growth and Oscillations of Solutions of Nonlinear Schrödinger Equation
}

\author{
Sergei B. Kuksin \\ Steklov Mathematical Institute, Vavilova St. 42, 117966 Moscow, Russia
}

Received: 20 May 1995/Accepted: 29 August 1995

To the memory of Natasha and Sergei Kozlov

\begin{abstract}
We study the nonlinear Schrödinger equation in an $n$-cube, $n=1,2,3$, under Dirichlet boundary conditions, treating it as a dynamical system in a function space formed by sufficiently smooth functions of $x$. We show that this space contains a distinguished small subset $\mathfrak{A}$ which is a recursion subset for the dynamical system and describe the dynamics of the equation in terms of the trajectory's recurrence to $\mathfrak{H}$. We use this description to estimate from below the space- and time-space oscillations of solutions in terms of a quantity, similar to the Reynolds number of classical hydrodynamics.
\end{abstract}

\section{Introduction}

We consider the nonlinear Schrödinger equation

$$
-i \dot{u}=\delta(-\Delta u+V(x) u)+|u|^{2} u, \quad u=u(t, x), \delta>0,
$$

with the space-variable $x$ in the $n$-cube $K^{n}=\left\{0 \leqq x_{j} \leqq \pi\right\}, n=1,2,3$, under Dirichlet boundary conditions

$$
\left.u\right|_{\partial K^{n}}=0 .
$$

We study the problem (1), (2) as a dynamical system in a function space $Z$ formed by sufficiently smooth complex functions $u(x)$,

$$
Z \subset C^{m}\left(K^{n} ; \mathbb{C}\right), \quad m \geqq 3,
$$

which vanish at $\partial K^{n}$. That is, given $u_{0} \in Z$ we interpret the solution $u(t, x)$ of (1), (2) with $u(0, x)=u_{0}(x)$ as a curve $u(t) \in Z$ and study the trajectories $u(t)$ as well as the flow-maps $S^{t}: Z \rightarrow Z, u_{0} \mapsto u(t)$.

The problem (1), (2) is well-known to be Hamiltonian with the Hamiltonian $\mathscr{H}$,

$$
\mathscr{H}(u(x))=\int\left(\frac{\delta}{2}|\nabla u(x)|^{2}+\frac{\delta}{2} V(x)|u(x)|^{2}+\frac{1}{4}|u(x)|^{4}\right) d x /(2 \pi)^{n},
$$


which is an integral of motion for the dynamical system:

$$
\text { const }=\mathscr{H}(u(t))=: E^{2},
$$

(see [7] and below).

In the space $Z$ we define the subset $\mathfrak{U}^{\delta}$,

$$
\mathfrak{A}^{\delta}=\left\{\left.u(x) \in Z|| u\right|_{\infty}<K \delta^{\mu}\|u\|^{1-2 \mu}\right\},
$$

where $|\cdot|_{\infty}$ is the $L^{\infty}$-norm, $\|\cdot\|$ is the norm in $Z, K$ is an absolute constant and

$$
\mu=\mu(m)=\frac{m(m-1)}{2 m^{2}+3 m-3}
$$

(so $\mu(3)=\frac{1}{4}$ and $\mu \nearrow \frac{1}{2}$ as $m$ grows).

For reasons we explain later, we call $\mathfrak{U}^{\delta}$ "the essential part of the phase-space $Z$." The set $\mathfrak{A}^{\delta}$ becomes small with $\delta$ and becomes relatively small as $\|u\|$ grows. Indeed, when $\delta \searrow 0$ the sets $\mathfrak{U}^{\delta}$ form a sequence of embedded domains with zero intersection, besides for $\delta$ fixed and for $S_{r}=\{\|u\|=r\}$ the intersection $\mathfrak{U}^{\delta} \cap S_{r}$ in the $L^{\infty}$-norm has diameter $\leqq 2 K \delta^{\mu} r^{1-2 \mu}$, thus forming a relatively small part of the sphere $S_{r}$ which has the $L^{\infty}$-diameter $\geqq C^{-1} r$.

For $m$ large we have $\mu \approx 1 / 2,1-2 \mu \approx 0$ and in a finite part of the space $Z$ the set $\mathfrak{U}^{\delta}$ looks like a tube of the $L_{\infty}$-diameter $\sim K \sqrt{\delta}$.

In parts 2,3 we show that - in a sense - dynamics of (1), (2) outside $\mathfrak{A}^{\delta}$ is simple and the whole dynamics of $(1),(2)$ is determined by its rather complicated behavior in $\mathfrak{A}^{\delta}$. More exactly, we prove

Theorem. If $u(t, x)$ is a solution of (1), (2) with $u(0, x)=u_{0} \notin \mathfrak{A}^{\delta},\left\|u_{0}\right\|=r$, then there exist $t^{\prime}, t^{\prime \prime} \leqq C r^{-a} \delta^{-b}$ such that $\left\|u\left(t^{\prime}\right)\right\|=2 r$ and $u\left(t^{\prime \prime}\right) \in \mathfrak{U}^{\delta}$. The positive numbers $a, b, C$ are $\delta, r$-independent.

Due to the theorem, a trajectory of (1), (2) either

i) moves outside $\mathfrak{U}^{\delta}$ toward this set, finally entering $\mathfrak{U}^{\delta}$ and increasing its $Z$-norm (at least doubling it),

or

ii) moves inside $\mathfrak{A}^{\delta}$.

Since the hamiltonian $\mathscr{H}$ is an integral of motion, then the growth of the "smooth" Z-norm at the stage i) means that low Fourier modes (in $x$ ) of the solution decrease while high modes increase - energy of this solution goes to high frequencies (the phenomenon also known as the direct cascade of energy, see more on the subject in $[11,8,9]$ ). Very likely during the stage ii) the $Z$-norm decreases (the energy goes to low modes - the inverse cascade of energy) and finally the solution leaves $\mathfrak{U}^{\delta}$, if $E$ in (5) is sufficiently large (more precisely, if large is the ratio $E / \delta$ ). This is certainly the case for solutions of the Zakharov-Shabat equation (Eq. (1) with $V \equiv 0, n=1$ ) which start outside $\mathfrak{A}^{\delta}$ since this equation is integrable and all its solutions are almost-periodic in time; they must decrease the $Z$-norm somewhere, so - inside $\mathfrak{A}^{\delta}$.

In [4] Ju.S. Ilyashenko obtained a description of dynamics of the KuramotoSivashinsky equation, similar to i), ii). Since that equation is parabolic, its solutions tend to a bounded finite-dimensional attractor in the corresponding function phasespace. On its way to the attractor each trajectory changes from i) to ii) a finite 
number of times and ends at the stage ii) in the vicinity of the attractor (see [4], especially Part 2.2).

Since $\|u\| \geqq K^{1 /(2 \mu)} \sqrt{\delta}$ for each $u \notin \mathfrak{U}^{\delta}$, then by the time

$$
T_{\text {pull }}=C_{1} \delta^{-b-a / 2}, 1
$$

the flow $\left\{S^{t}\right\}$ will pull the whole space $Z$ through a narrow slit formed by the set $\mathfrak{U}^{\delta}$. Phenomenons of this kind are typical for Hamiltonian partial differential equations considered in smooth function spaces [8]. For deep symplectic reasons they are impossible in some distinguished function phase-spaces of low smoothness (see $[8,9]$ and references therein).

By the theorem a solution $u(t)$ of (1), (2) either is in $\mathfrak{U}^{\delta}$, or it moves fast toward $\mathfrak{U}^{\delta}$. In Part 4 we use this description of the dynamics of $(1),(2)$ to estimate oscillations of its solutions $u(t, x)$. As a measure of the oscillations we propose the function

$$
\omega_{m}(t)=\|u(t)\| /|u(t)|_{\infty} .
$$

(Due to (3) this is something like the $C^{m}$-norm of the solution divided by its $L^{\infty}$-norm). The theorem implies that $\omega_{m}(t)$ becomes large at some point $t_{*}$ of each time-interval of the length $l$,

$$
l=C / \sqrt{E \delta}
$$

( $E$ was defined in $(5)$; the factor $\delta^{-1 / 2}$ corresponds to a natural time-scaling, see Part 4). Here "large" means that

$$
\omega_{m}\left(t_{*}\right) \geqq C^{-1}(E / \delta)^{\kappa}
$$

with some $\kappa>1 / 5$.

We also consider a quantity which takes into account time-oscillations of solutions. We define the function $\Omega_{m}(t)$ as

$$
\Omega_{m}=\frac{\|u(t)\|+C\|\dot{u}(t)\| / \sqrt{E \delta}}{|u(t)|_{\infty}}
$$

and prove that averaging of $\Omega_{m}$ along each time-interval of length $\geqq 3 l$ is at least one-sixth of the r.h.s. of (6). Roughly,

$$
\left\langle\Omega_{m}\right\rangle_{\mathrm{loc}} \geqq C^{-1}(E / \delta)^{\kappa}
$$

(here $\langle\cdot\rangle_{\text {loc }}$ stands for local averaging in $t$ ).

Thus, solutions of $(1),(2)$ oscillate at least as $(E / \delta)$ in a positive degree. We suppose that for (1), (2) (and other Hamiltonian PDE's) the quantity $E / \delta$ plays a role similar to the role of the Reynolds number for the equations of hydrodynamics [MY, Chapter 1].

The theorem describes behavior of solutions of the problem (1), (2) for $0 \leqq T_{\text {pull }}$ ( since $t^{\prime}, t^{\prime \prime} \leqq T_{\text {pull }}$ ) and gives no information on long-time behavior of individual solutions. Based on the fast ${ }^{2}$ growth of the norms $\|u(t)\|$ of solutions outside $\mathfrak{A}^{\delta}$ one could conjecture that the solutions grow fast (at least - grow indefinitely) as $t$ grows. This guess fails since the problem (1), (2) with $n=1, V \equiv 0$ is integrable all its solutions are almost-periodic in time (and so are bounded). One could try

$1=C_{1} \delta^{-1}$ since $a+b / 2=1-$ see in Part 3 .

2 In fact - super exponentially fast. 
to save the conjecture imposing the additional restriction that the Eq. (1) must be "typical" (and so non integrable), but this also does not help since for $n=1,2$ and for typical $V(x)$ the problem (1), (2) has abundance of time-quasiperiodic solutions (see [7] for $n=1$ and [3] for $n=2$ ). These solutions jointly are dense near zero and their $Z$-norms jointly are unbounded (cf. Proposition in the introduction of [8]). It is an interesting open problem if the problem (1), (2) with $n \geqq 2$ has unbounded (in $Z$ ) solutions.

We remark that even in the integrable case $(V=0, n=1)$ the Eq. (1) with small $\delta$ is rather complicated. Limiting (as $\delta$ goes to zero) behaviour of its solutions is studied in [5]; detailed analysis of solutions with fixed $0<\delta \ll 1$ was not done yet. $^{3}$

A few words on the notations we use: by $C, C_{1}$ etc. we denote different positive constants, independent of the main parameters (like $\delta$ and $E$ ), by $\|\cdot\|_{s}$ denote the $C^{s}$-norms, by $|\cdot|_{p}$ - the $L^{p}$-norms. We write a function $u(t, x)$ as $u(t)$ when we treat it as a curve in a function space of functions of $x$.

\section{The Equation and its Phase-Space}

We study the nonlinear Schrödinger equation in the $n$-cube $K^{n}=\left\{x \in \mathbb{R}^{n} \mid 0 \leqq x_{j} \leqq \pi\right\}$, $n=1,2,3$, under Dirichlet boundary conditions:

$$
\begin{gathered}
-i \dot{u}=\delta(-\Delta u+V(x) u)+|u|^{2} u, \quad u=u(t, x), x \in K^{n}, \\
\left.u\right|_{\partial K^{n}}=0,
\end{gathered}
$$

where $0<\delta \leqq 1$ and $V$ is a smooth real potential. It is convenient to extend $V(x)$ to an even $2 \pi$-periodic function $V(x), x \in T^{n}=\mathbb{R}^{n} / 2 \pi \mathbb{Z}^{n}$,

$$
V\left(x_{1}, \ldots, x_{n}\right)=V\left(x_{1}, \ldots,-x_{j}, \ldots, x_{n}\right), \quad j=1, \ldots, n,
$$

and extend solution $u(t, x)$ to an odd $2 \pi$-periodic function $u(t, x), x \in T^{n}$,

$$
u\left(x_{1}, \ldots, x_{n}\right)=-u\left(x_{1}, \ldots,-x_{j}, \ldots, x_{n}\right), \quad j=1, \ldots, n,
$$

thus recasting (1.2) as odd periodic boundary conditions (1.3). ${ }^{4}$

We suppose that the potential $V(x)$ as a function on the torus $T^{n}$ is even and smooth,

$$
V \in C^{\infty}\left(T^{n}, \mathbb{R}\right),
$$

and denote by $A$ the linear operator

$$
A(u(x))=-\Delta u+V(x) u .
$$

Let us take any Banach space $H$ of complex functions $u(x), x \in T^{n}$, with the norm $\|\cdot\|$, which is a Banach algebra with involution (i.e., $\|\bar{u}\|=\|u\|$ and $\|u v\| \leqq$ $C\|u\|\|v\|)$. Suppose that $H$ is embedded to some space $C^{m}=C^{m}\left(T^{n}, \mathbb{C}\right), m \geqq 3$ :

$$
H \subset C^{m}, \quad\|u\|_{m} \leqq\|u\| .
$$

3 Investigation of finite-gap solutions of (1) $(V=0, n=1)$ with small $\delta$ is interesting since there are good hopes that using the KAM-theorems [7] one can study solutions of (1) with nonzero potential $V$ and large values of $E / \delta$ as perturbations of the finite-gap trajectories.

${ }^{4}$ Clearly $u(x)\left(x \in T^{n}\right)$ as in (1.3) meets (1.2) being restricted to $K^{n}$. 
Suppose also that $H$ is invariant for the flow of (1.1) (solution of (1.1) with initial data from $H$ stays in $H$ for all $t$ ). Then the subspace $Z \subset H$ formed by odd functions,

$$
Z=\{u(x) \in H \mid u \text { meets }(1.3)\}
$$

also is invariant. We equip $Z$ with the norm $\|\cdot\|$ and take it for the phase space of the problem (1.1), (1.3).

Example $1(n=1)$. Since the $L_{2}$-norm and the Hamiltonian $\mathscr{H}$ (see below) are integrals of motion for Eq. (1.1), then the Sobolev $H^{1}$-norm (in $x$ ) of a solution $u(t, x)$ can be estimated via the $H^{1}$-norm of the initial data $u(0, x)$ uniformly in $t$. Therefore, Eq. $(1.1)$ is well-posed in the space $H^{1}\left(T^{1} ; \mathbb{C}\right)$. Simple induction shows that it is also well-posed in the spaces $H^{l}\left(T^{1} ; \mathbb{C}\right), l \geqq 1$. Since $H^{l} \subset C^{m}$ if $l>m+\frac{1}{2}$, then we can take

$$
H=H^{l}\left(T^{1} ; \mathbb{C}\right), \quad l>m+\frac{1}{2} .
$$

Example $2(n=2,3, V \equiv 0)$. Now the equation also is well-posed in the Sobolev spaces $H^{l}, l \geqq 1$ - this is a rather nontrivial result of J. Bourgain [2] - and we can take $H=H^{l}\left(\mathbb{T}^{n} ; \mathbb{C}\right)$, where

$$
l> \begin{cases}m+1, & n=2, \\ m+\frac{3}{2}, & n=3,\end{cases}
$$

since these spaces are embedded to $C^{m}\left(T^{n} ; \mathbb{C}\right)$. For $V$ nonzero see Part 5.4 below.

We supply the linear space $Z$ with the skew-symmetric two-form, defining the skew-product of functions $u(x), v(x) \in Z$ as

$$
-\operatorname{Im} \int u \bar{v} d x /(2 \pi)^{n}
$$

This two-form defines a constant-coefficient symplectic structure in the phase-space $Z$ and (1.1) becomes the Hamiltonian equation with the Hamiltonian $\mathscr{H}$,

$$
\mathscr{H}(u)=\int_{T^{n}}\left(\frac{\delta}{2}|\nabla u(x)|^{2}+\frac{\delta}{2} V(x)|u(x)|^{2}+\frac{1}{4}|u(x)|^{4}\right) d x /(2 \pi)^{n} .
$$

In particular, $\mathscr{H}(u(t, \cdot))=$ const for any solution $u$ of (1.1) in $Z$ (see e.g. in [7]).

Multiplying $(1.1)$ by $\bar{u}(t, x)$, integrating over $T^{n}$ and taking the imaginary part of the equality we get that

$$
|u(t, \cdot)|_{2}=\text { const }
$$

for any solution $u$.

We also observe that since $H \subset C^{m}$, then by the Gagliardo-Nirenberg inequality

$$
\|u\|_{k} \leqq C_{k}|u|_{\infty^{\frac{m-k}{m}}}^{\frac{m}{m}}\|u\|^{\frac{k}{m}}, \quad 0 \leqq k \leqq m
$$

(see [1]; the special case of the inequality we use now goes back to HadamardLandau-Kolmogorov).

\section{The Main Estimate for the Flow}

In this part, we study solutions $u(t) \in Z$ of (1.1) such that $u(0)=u_{0}$, where

$$
\left\|u_{0}\right\|=1, \quad\left|u_{0}\right|_{\infty}=\varrho
$$


and

$$
\varrho \geqq K \delta^{\mu} \quad \text { with } \quad \mu=\mu(m)=\frac{m(m-1)}{2 m^{2}+3 m-3}
$$

(clearly $\varrho \leqq 1$ ). The function $\mu$ increases with $m \geqq 3$ and

$$
\mu(3)=\frac{1}{4}, \quad \mu(m) \nearrow \frac{1}{2} \quad \text { as } m \longrightarrow \infty .
$$

We study the solutions for $0 \leqq t \leqq T$, where

$$
T=K_{1} \varrho^{-\frac{2 m+1}{m}} \leqq K_{1} K^{-\frac{2 m+1}{m}} \delta^{-v}, \quad v=\frac{2 m+1}{m} \mu .
$$

The $m$-independent positive constants $K, K_{1}$ are such that

$$
K \geqq 2 \sqrt{C_{+} K_{1}}, \quad K \geqq 2,
$$

where $C_{+}$is an absolute constant from the inequality (2.8) below. The values of $K, K_{1}$ will be specified later.

We shall show that each solution of (1.1), (2.1) doubles its $Z$-norm somewhere on the time-segment $[0, T]$. To prove the doubling we suppose that on the contrary

$$
\|u(t)\| \leqq 2 \text { for all } 0 \leqq t \leqq T,
$$

and shall extract a contradiction from this assumption.

Below by $C, C_{1}$, etc., we denote different positive constants independent of $\delta, \varrho$ and $K, K_{1}$ (which can depend on $m$ ).

Elementary calculations show that for $T$ as above we have

$$
T \leqq K_{2} \varrho^{2 / m} \delta^{-1}, \quad K_{2}=K_{1} K^{-\frac{2 m+3}{m}}<\frac{1}{4 C_{+}} .
$$

Suppose that for some $T_{1} \leqq T$ we have

$$
|u(t)|_{\infty} \leqq 2 \varrho \quad \text { if } 0 \leqq t \leqq T_{1} .
$$

Then by (2.5) and (1.5),

$$
|A u(t)|_{\infty} \leqq C\|u(t)\|_{2} \leqq C_{+}|u(t)|_{\infty}^{\frac{m-2}{m}} \leqq 2 C_{+} \varrho^{\frac{m-2}{m}} .
$$

Take any $x \in T^{n}$. Multiplying $(1.1)$ by $\bar{u}(t, x)$ and taking the imaginary part we get

$$
\left.\left|\frac{1}{2} \frac{d}{d t}\right| u(t, x)\right|^{2}|\leqq \delta| A u(t, x)|| u(t, x) \mid \leqq 2 \delta C_{+} \varrho^{2-\frac{2}{m}} .
$$

In particular, $|u(t, x)|^{2} \leqq \varrho^{2}+4 t \delta C_{+} \varrho^{2-\frac{2}{m}}$ and using (2.6) we see that

$$
|u(t, x)|^{2} \leqq \varrho^{2}\left(1+4 K_{2} C_{+}\right)<2 \varrho^{2} .
$$

It means that for each solution which satisfies (2.1), (2.5) the estimate (2.7) holds with $T_{1}=T$ :

$$
|u(t)|_{\infty} \leqq 2 \varrho \text { if } 0 \leqq t \leqq T .
$$

Now let us denote

$$
w(t, x)=|u(t, x)|^{2}-\left|u_{0}(x)\right|^{2} .
$$


Then $w(0, x) \equiv 0$ and by $(2.9)$,

$$
\left|\frac{d}{d t} w(t, x)\right| \leqq C \delta \varrho^{2-\frac{2}{m}} .
$$

Therefore

$$
|w(t)|_{\infty} \leqq C t \delta \varrho^{2-\frac{2}{m}} .
$$

Since $H$ is a Banach algebra, then by $(2.5),\|w(t)\| \leqq C$ for all $0 \leqq t \leqq T$. Now by (1.5) and (2.11),

$$
\|w(t)\|_{k} \leqq C\left(t \delta \varrho^{2-\frac{2}{m}}\right)^{\frac{m-k}{m}} .
$$

We rewrite (1.1) as

$$
\dot{u}-i\left(\left|u_{0}\right|^{2}+w\right) u=i \delta A u .
$$

Treating this equation as a nonautonomous linear ordinary differential equation for the complex function $t \mapsto u(t, x)$ with the right-hand side $i \delta A u$, we write its solution $u$ as

$$
\begin{aligned}
u(t, x)= & i \delta \int_{0}^{t} \exp \left(i(t-\tau)\left|u_{0}\right|^{2}+i \int_{\tau}^{t} w(\theta, x) d \theta\right) A u(\tau, x) d \tau \\
& +\exp \left(i t\left|u_{0}\right|^{2}\right) \exp \left(i \int_{0}^{t} w(\tau, x) d \tau\right) u_{0}(x)=: u_{1}+u_{2}
\end{aligned}
$$

( $u_{1}$ denotes the first summand and $u_{2}$ - the second). We wish to estimate $\|u(t)\|_{1}$ from below. To do so we shall estimate $\left\|u_{1}(t)\right\|_{1}$ from above and $\left\|u_{2}(t)\right\|_{1}$ from below.

Since both the functions $\left|u_{0}(x)\right|^{2}$ and $w(\theta, x)$ are real, then the norm of the exponential factor under the integral in $u_{1}$ equals one. Any first-order $x$-derivative of $u_{1}$ contains two terms (since we differentiate either $A u$ or the exponent). By (1.5) and (2.10),

$$
\|u(t)\|_{k} \leqq C \varrho^{\frac{m-k}{m}} \quad \text { for } 0 \leqq k \leqq m .
$$

As $\|A u\|_{k} \leqq C_{k}\|u\|_{k+2}$, then from (2.13) with $k=2,3$ we have

$$
\left\|u_{1}(t)\right\|_{1} \leqq C \delta \int_{0}^{t}\left((t-\tau)\left(\left\|u_{0} \bar{u}_{0}\right\|_{1}+\sup _{\theta \leqq t}\|w(\theta)\|_{1}\right) \varrho^{\frac{m-2}{m}}\right) d \tau+C \delta \varrho^{\frac{m-3}{m}} t .
$$

By (2.13) with $k=1$ and (2.12), (2.5), $\left\|u_{0} \bar{u}_{0}\right\|_{1}+\|w(\theta)\|_{1} \leqq C \varrho^{2 \frac{m-1}{m}}$. Therefore

$$
\left\|u_{1}(t)\right\|_{1} \leqq C \delta \varrho^{\frac{3 m-4}{m}} t^{2}+C \delta \varrho^{\frac{m-3}{m}} t
$$

Now we pass to the function $u_{2}(t, x)$ and write it as $u_{2}=u_{2}^{1} u_{2}^{2} u_{0}$, where

Clearly

$$
u_{2}^{1}=\exp \left(i t\left|u_{0}\right|^{2}\right), \quad u_{2}^{2}=\exp i \int_{0}^{t} w(\tau, x) d \tau \text {. }
$$

$$
\left\|u_{2}\right\|_{1} \geqq \sup _{x}\left|\left(\nabla_{x} u_{2}^{1}\right) u_{2}^{2} u_{0}\right|-\left\|u_{2}^{2}\right\|_{1}\left|u_{2}^{1}\right|_{\infty}\left|u_{0}\right|_{\infty}-\left\|u_{0}\right\|_{1}\left|u_{2}^{2}\right|_{\infty}\left|u_{2}^{1}\right|_{\infty} .
$$

We shall estimate the three terms in the r.h.s. As $\left|u_{2}^{1}\right| \equiv 1,\left|u_{2}^{2}\right| \equiv 1$, then we already know that

$$
\left|u_{2}^{1}\right|_{\infty}=1, \quad\left|u_{0}\right|_{\infty}=\varrho, \quad\left|u_{2}^{2}\right|_{\infty}=1, \quad\left\|u_{0}\right\|_{1} \leqq C \varrho^{\frac{m-1}{m}} .
$$


Since $\left|u_{2}^{2}\right| \equiv 1$, then

$$
\sup _{x}\left|\left(\nabla_{x} u_{2}^{1}\right) u_{2}^{2} u_{0}\right|=\left.t \sup _{x}\left|u_{0}(x) \nabla_{x}\right| u_{0}\right|^{2}\left|=\frac{2}{3} t \sup _{x}\right| \nabla_{x}\left|u_{0}\right|^{3} \mid \geqq t C^{-1} \varrho^{3},
$$

where the last estimate follows from (2.1) since $u_{0}$ vanishes at $\partial K^{n}$.

It remains to estimate $\left\|u_{2}^{2}\right\|_{1}$. Using (2.12) we get that

$$
\left\|u_{2}^{2}\right\|_{1} \leqq t \sup _{0 \leqq \tau \leqq t}\|w(\tau)\|_{1} \leqq C t\left(t \delta \varrho^{2-\frac{2}{m}}\right)^{\frac{m-1}{m}} .
$$
below:

Now the estimates (2.14)-(2.18) jointly imply the estimate for $u(t, x)$ from

$$
\begin{aligned}
\|u(t)\|_{1} \geqq & C_{1}^{-1} t \varrho^{3}-C_{2} t^{2} \delta \varrho^{\frac{3 m-4}{m}}-C_{3} t \delta \varrho^{\frac{m-3}{m}} \\
& -C_{4} t^{\frac{2 m-1}{m}} \varrho^{1+2\left(\frac{m-1}{m}\right)^{2}} \delta^{\frac{m-1}{m}}-C_{5} \varrho^{\frac{m-1}{m}} .
\end{aligned}
$$

By (2.13) we have

$$
\|u(t)\|_{1} \leqq C_{*} \varrho^{\frac{m-1}{m}} \quad \text { for } 0 \leqq t \leqq T .
$$

On the other hand, by (2.19),

$$
\left\|u\left(t_{*}\right)\right\|_{1} \geqq 2 C_{*} \varrho^{\frac{m-1}{m}},
$$

if $t_{*}$ meets the following system of inequalities:

$$
\left\{\begin{array}{l}
C_{1}^{-1} t \varrho^{3} \geqq\left(5 C_{*}+C_{5}\right) \varrho^{\frac{m-1}{m}}, \\
C_{2} t^{2} \delta \varrho^{\frac{3 m-4}{m}}<C_{*} \varrho^{\frac{m-1}{m}}, \\
C_{3} t \delta \varrho^{\frac{m-3}{m}}<C_{*} \varrho^{\frac{m-1}{m}}, \\
C_{4} t^{\frac{2 m-1}{m}} \varrho^{1+2\left(\frac{m-1}{m}\right)^{2}} \delta^{\frac{m-1}{m}}<C_{*} \varrho^{\frac{m-1}{m}},
\end{array}\right.
$$

or

$$
\left\{\begin{array}{l}
t \geqq C_{1}^{\prime} \varrho^{-2-\frac{1}{m}} \\
t<C_{2}^{\prime} \delta^{-\frac{1}{2}} \varrho^{-\frac{2 m-3}{2 m}}, \\
t<C_{3}^{\prime} \delta^{-1} \varrho^{\frac{2}{m}} \\
t<C_{4}^{\prime} \delta^{-\frac{m-1}{2 m-1}} \varrho^{-\frac{2 m^{2}-3 m+2}{m(2 m-1)}} .
\end{array}\right.
$$

The first two inequalities in (2.21) are consistent if

$$
\varrho>C_{1}^{\prime \prime} \delta^{\frac{m}{2 m+5}} ;
$$

the first and the third are if

$$
\varrho>C_{2}^{\prime \prime} \delta^{\frac{m}{2 m+3}} ;
$$

and the first and the fourth are if

$$
\varrho>C_{3}^{\prime \prime} \delta^{\mu}, \quad \mu=\mu(m) \text { as in (2.2). }
$$

Let us denote $\tilde{C}=\max \left\{C_{1}^{\prime \prime}, C_{2}^{\prime \prime}, C_{3}^{\prime \prime}\right\}$. Then the system $(2.21)$ is consistent if

$$
\varrho>\tilde{C} \delta^{\mu} \text {. }
$$


We choose in (2.3) $K_{1}=C_{1}^{\prime}$ and take for $K$ any number such that

$$
K>\tilde{C}, \quad K \geqq 2, \quad K \geqq 2 \sqrt{C_{+} K_{1}} .
$$

With this choice of $K, K_{1}$ the assumptions (2.4) are met and $t=T:=K_{1} \varrho^{-2-1 / m}$ satisfies (2.21). Thus, $\|u(T)\|_{1} \geqq 2 C_{*} \varrho^{(m-1) / m}$ in a contradiction with (2.20).

Since the assumption (2.5) led to a contradiction, then the solution $u(t)$ doubles its norm for some $t \leqq T=K_{1} \varrho^{-(2 m+1) / m}$ with $K_{1}$ as above and we get

Theorem 1. There exist constants $K, K_{1}$ such that if $u(t)$ is a solution of (1.1) and $u(0)=u_{0}$ satisfies $(2.1)$ with some

$$
\varrho \geqq K \delta^{\mu}, \quad \mu=\mu(m)=\frac{m(m-1)}{2 m^{2}+3 m-3},
$$

then there exists $t_{1}$,

$$
t_{1} \leqq K_{1} \varrho^{-2-1 / m} \leqq C \delta^{-v}, \quad v=\frac{(2 m+1)(m-1)}{2 m^{2}+3 m-3}<1
$$

such that $\left\|u\left(t_{1}\right)\right\| \geqq 2$.

Remark 1. The statement and its proof remain true if we replace $\mu(m)$ and $v(m)$ by any $\tilde{\mu}, \tilde{v}$ such that $0<\tilde{\mu} \leqq \mu(m), \tilde{v}=\frac{2 m+1}{m} \tilde{\mu}$.

\section{Rescaled Estimates and the Essential Part of the Phase-Space}

We define a subset $\mathfrak{U} \subset Z$ which we call "essential part of the phase-space $Z$ " as follows:

$$
\mathfrak{A}=\left\{\left.u(x) \in Z|| u\right|_{\infty}<K \delta^{\mu}\|u\|^{1-2 \mu}\right\},
$$

with $\mu$ and $K$ as in Theorem 1. Since $|u|_{\infty} \leqq\|u\|=(\|u\| / \sqrt{\delta})^{2 \mu} \delta^{\mu}\|u\|^{1-2 \mu}$, then $\mathfrak{U}$ contains the ball

$$
\left\{u \in Z \mid\|u\|<\sqrt{\delta} K^{1 /(2 \mu)}\right\}
$$

(and $\mathfrak{U}$ does not contain some points of the norm $C \sqrt{\delta}$, where $C>K^{1 /(2 \mu)}$ is $\delta$-independent).

Now let us consider a solution of (1.1) with any initial condition outside $\mathfrak{A}$ :

$$
u(0, x)=u_{0}(x) \notin \mathfrak{A} .
$$

We denote $r=\left\|u_{0}\right\|, v=u / r$. Then

$$
v(0, x)=v_{0}(x)=u_{0} / r, \quad\left\|v_{0}\right\|=1,
$$

and

$$
-i v=\delta A v+r^{2}|v|^{2} v .
$$

So if we stretch the time $t$ as

$$
t=r^{-2} \tau,
$$

then we get for $v$ the rescaled equation:

$$
-i v_{\tau}^{\prime}=\delta_{1} A v+|v|^{2} v, \quad \delta_{1}=\delta / r^{2} .
$$


Since $u_{0} \notin \mathfrak{A}$, then by (3.1) $r \geqq \sqrt{\delta} K^{1 /(2 \mu)}>\sqrt{\delta}$. So

$$
\delta_{1} \leqq 1 \quad \text { and } \quad\left\|v_{0}\right\|=1, \quad\left|v_{0}\right|_{\infty}=\left|u_{0}\right|_{\infty} / r \geqq K\left(\delta / r^{2}\right)^{\mu}=K \delta_{1}^{\mu} .
$$

Now application of Theorem 1 with $\delta=\delta_{1}$ to the problem (3.2), (3.3) implies existence of $\tau_{1} \leqq C \delta_{1}^{-v}$, corresponding to $t_{1}=r^{-2} \tau_{1} \leqq C r^{-2}\left(\delta / r^{2}\right)^{-v}$, such that

$$
r_{1}:=\left\|u\left(t_{1}\right)\right\| \geqq 2 r=2\left\|u_{0}\right\| .
$$

If still $u\left(t_{1}\right) \notin \mathfrak{U}$, then we can iterate the procedure to find $t_{2} \leqq C r_{1}^{-2}\left(\delta / r_{1}^{2}\right)^{-v}$ such that

$$
r_{2}:=\left\|u\left(t_{1}+t_{2}\right)\right\| \geqq 2^{2}\left\|u_{0}\right\| .
$$

We can iterate further, provided that $u(t) \notin \mathfrak{A}$, to find $t_{1}, \ldots, t_{M}$ such that

$$
r_{M}:=\left\|u\left(t_{1}+\cdots+t_{M}\right)\right\| \geqq 2^{M}\left\|u_{0}\right\|
$$

and

$$
t_{M} \leqq C r_{M-1}^{-2}\left(\delta / r_{M-1}^{2}\right)^{-v} .
$$

Denote $u_{M}=u\left(t_{1}+\cdots+t_{M}\right)$. Since the flow of (1.1) preserves the Hamiltonian $\mathscr{H}$, then

$$
\left|u_{M}\right|_{4} \leqq 4 \mathscr{H}\left(u_{M}\right)=4 \mathscr{H}\left(u_{0}\right) .
$$

By Sobolev's theorem and the Gagliardo-Nirenberg inequality (see [1]),

$$
\left|u_{M}\right|_{\infty} \leqq C\left\|u_{M}\right\|_{1,4} \leqq C_{1}\left\|u_{M}\right\|^{\frac{1}{m}}\left|u_{M}\right|_{4}^{\frac{m-1}{m}} \leqq C r_{M}^{\frac{1}{m}},
$$

where $\|\cdot\|_{1,4}$ stands for the norm in the Sobolev space $W^{1,4}\left(T^{n} ; \mathbb{C}\right.$ ) (we recall that $n \leqq 3$ ). If $u_{M} \notin \mathfrak{A}$, then we have $\left|u_{M}\right|_{\infty} \geqq K \delta^{\mu} r_{M}^{1-2 \mu}$. Using (3.5) we find that

$$
C \geqq K \delta^{\mu} r_{M}^{1-2 \mu-1 / m} \text {. }
$$

Suppose for a moment that $n=2$ or 3 . Then $1-2 \mu>1 / m$ and we see that the norms $r_{M}$ are bounded by an $M$-independent constant. Now (3.4) implies that $M \leqq M\left(u_{0}\right)$. It means that after a finite number of steps the solution $u(t)$ hits to $\mathfrak{H}$. The total time the solution $u(t)$ spends outside $\mathfrak{A}$ is estimated:

$$
\begin{aligned}
t_{1}+\cdots+t_{M} & \leqq C \delta^{-v}\left(r^{2 v-2}+r_{1}^{2 v-2}+\cdots\right) \\
& \leqq C \delta^{-v} r^{2 v-2}\left(1+4^{v-1}+4^{2(v-1)}+\cdots\right) .
\end{aligned}
$$

Since $v=1-\frac{5}{2 m}+O\left(m^{-2}\right)<1$, then

$$
t_{1}+\cdots+t_{M} \leqq m C \delta^{-v} r^{2 v-2} .
$$

If $n=1$, then $\left|u_{M}\right| \leqq C\left\|u_{M}\right\|_{2 / 3,2} \leqq C_{1}\left\|u_{M}\right\|^{2 /(3 m)}\left|u_{M}\right|_{2}^{(3 m-2) /(3 m)} \leqq C r_{M}^{2 /(3 m)}$ and (3.6) also follows.

We have proved the following result:

Theorem 2. If $u(t, x)$ is a solution of (1.1) with $u(0, x)=u_{0}(x) \notin \mathfrak{A},\left\|u_{0}\right\|=r$, then

1) there exists $t^{\prime} \leqq C_{1} r^{2 v-2} \delta^{-v}$ such that $\left\|u\left(t^{\prime}\right)\right\| \geqq 2 r$,

2) there exists $t^{\prime \prime} \leqq C_{2} r^{2 v-2} \delta^{-v}$ such that $u\left(t^{\prime \prime}\right) \in \mathfrak{A}$.

Here $v=v(m)$ as in Theorem 1. 
Remark 2. The arguments we used to prove Theorems 1,2 are applicable as well to negative times $t<0$. Thus, we can find negative $-t_{1}^{\prime},-t_{1}^{\prime \prime}$ such that $t_{1}^{\prime}, t_{1}^{\prime \prime}$ meet the same estimates as $t^{\prime}, t^{\prime \prime}$ and $\left\|u\left(-t_{1}^{\prime}\right)\right\| \geqq 2 r, u\left(-t_{1}^{\prime \prime}\right) \in \mathfrak{A}$.

In particular, $\mathfrak{U}$ is a recursion subset of the phase-space $Z$ - each trajectory of (1.1) in $Z$ visits $\mathfrak{A}$ at arbitrarily large values of times.

In addition, since by (3.1) $r \geqq \sqrt{\delta} K^{1 /(2 \mu)}$ for each $u_{0} \notin \mathfrak{A}$, then $t^{\prime \prime} \leqq C \delta^{-1}$ and by the time

$$
T_{\text {pull }}=C \delta^{-1}
$$

the flow $\left\{S^{t}\right\}$ will pull the whole space $Z$ through its essential part $\mathfrak{A}$.

\section{Oscillations of Solutions}

In this part we discuss properties of solutions $u(t)$ with large values of the quantity $R$,

$$
R=\frac{E}{\delta} \quad \text { where } \mathscr{H}(u(t)) \equiv E^{2} .
$$

(We denote the ratio by $R$ to note its similarity with the Reynolds number of classical hydrodynamics [10].) To do so, it is convenient to introduce the "fast time" $\tau=\sqrt{\delta} t$, so

and to write (1.1) as

$$
\frac{\partial}{\partial t}=\sqrt{\delta} \frac{\partial}{\partial \tau}
$$

$$
-i \sqrt{\delta} u_{\tau}^{\prime}=-\delta \Delta u+|u|^{2} u
$$

Our goal is to study the function

$$
\omega(\tau)=\omega_{m}(\tau)=\frac{\|u(\tau)\|}{|u(\tau)|_{\infty}},
$$

which we propose to use as a measure of the oscillating solutions of (4.1). (The subindex $m$ recalls that $H \subset C^{m}$ - we treat $\|\cdot\|$ as an "almost $C^{m}$-norm.") We shall show that Theorem 2 implies estimates for averaged characteristics of $\omega_{m}$ in terms of $R$.

Consider any solution $u(\tau) \in Z, 0 \leqq \tau \leqq T$, of (4.1) such that $\mathscr{H}(u(\tau)) \equiv E^{2}$, where $E$ is bounded from below by a positive $\delta$-independent constant, $E \geqq C^{-1}$. Let us denote $\|u(\tau)\|=r(\tau)$. Since

$$
C^{-1} \leqq E^{2}=\frac{\delta}{2}|\nabla u|_{2}^{2}+\frac{1}{4}|u|_{4}^{4} \leqq \frac{\delta n}{2} r^{2}+\frac{1}{4} r^{4},
$$

then

Let us denote

$$
r(\tau)^{2} \geqq E / C_{1}
$$

Then by (4.2),

$$
\tilde{\Delta}(\tau)=r(\tau)^{2(v-1)} \delta^{-v+1 / 2} .
$$

$$
\tilde{\Delta} \leqq C_{2} \Delta, \quad \Delta=E^{v-1} \delta^{-v+1 / 2},
$$

and by Theorem 2 if $u\left(\tau_{0}\right) \notin \mathfrak{A}$, then

$$
\begin{gathered}
\left\|u\left(\tau_{0}+\tau_{1}\right)\right\|=2\left\|u\left(\tau_{0}\right)\right\| \text { for some } \tau_{1} \leqq C_{1} \tilde{\Delta} \leqq C^{\prime} \Delta, \\
u\left(\tau_{0}+\tau_{2}\right) \in \mathfrak{U} \quad \text { for some } \tau_{2} \leqq C_{2} \tilde{\Delta} \leq C \Delta .
\end{gathered}
$$


Since for $u(\tau) \in \mathfrak{U}$ we have

$$
\omega_{m}(\tau) \geqq K^{-1}\left(r^{2} / \delta\right)^{\mu} \geqq C_{1}^{-1} R^{\mu}
$$

(we use (4.2)), then by (4.4) we get

Proposition 1. For each solution as above we have

$$
\sup _{\tau_{1} \leqq \tau \leqq \tau_{1}+C \Delta} \omega_{m}(\tau) \geqq C_{1}^{-1} R^{\mu(m)}
$$

if $0 \leqq \tau_{1} \leqq T-C \Delta$.

We recall that $\mu=\mu(m) \geqq 1 / 4$ tends to $1 / 2$ as $m \rightarrow \infty$.

Next we estimate an average of the function

and start with

$$
\widetilde{\Omega}_{m}=\omega_{m}+C \Delta\left|\omega_{m}^{\prime}\right|
$$

Lemma. If $u\left(\tau_{0}\right) \in \overline{\mathfrak{U}}$ is such that $u\left(\tau_{0}+\varepsilon\right) \notin \mathfrak{U}$ for all sufficiently small $\varepsilon>0$, then there exists $\tau_{*}>\tau_{0}, \tau_{*} \leqq \tau_{0}+C \Delta$, such that $u\left(\tau_{*}\right) \in \mathfrak{U}$ and

$$
\frac{1}{\tau_{*}-\tau_{0}} \int_{\tau_{0}}^{t_{*}} \widetilde{\Omega}_{m}(\tau) d \tau \geqq \omega_{m}\left(\tau_{0}\right) .
$$

Proof. Since the set $\mathfrak{A}$ is open, then $u\left(\tau_{0}\right) \notin \mathfrak{U}$ and we can find $\tau^{\prime}=\tau_{1}$ as in (4.3). We can suppose that $\|u(\tau)\|<2\left\|u\left(\tau_{0}\right)\right\|$ for $\tau_{0} \leqq \tau<\tau^{\prime}$. Then by the estimate (2.10) from the proof of Theorem $1,\left|u\left(\tau^{\prime}\right)\right|_{\infty} \leqq 2\left|u\left(\tau_{0}\right)\right|_{\infty}$. Therefore $\omega\left(\tau^{\prime}\right) \geqq$ $\omega\left(\tau_{0}\right)$. If $\tau^{\prime} \in \mathfrak{A}$ then at the moment $\tau^{\prime \prime}$ of the next doubling of the $H$-norm we also have $\omega\left(\tau^{\prime \prime}\right) \geqq \omega\left(\tau^{\prime}\right) \geqq \omega\left(\tau_{0}\right)$. Finally, due to the proof of the second statement of Theorem 2, we shall find a point $\tau_{*}=\tau^{(N)} \in \mathfrak{U}$ such that $\omega\left(\tau_{*}\right) \geqq \omega\left(\tau_{0}\right)$ and $\tau_{*} \in\left[\tau_{0}, \tau_{0}+C \Delta\right]$.

Let us denote

$$
\theta=\sup \left\{\tau \in\left[\tau_{0}, \tau_{*}\right] \mid \omega(\tau) \leqq \omega\left(\tau_{0}\right)\right\} .
$$

Then $\omega(\theta)=\omega\left(\tau_{0}\right)$ and $\omega \geqq \omega\left(\tau_{0}\right)$ everywhere in $\left[\theta, \tau_{*}\right]$. Therefore denoting $f(\tau)=\omega(\tau)-\omega\left(\tau_{0}\right)$ we get:

$$
\begin{aligned}
\frac{1}{\theta-\tau_{0}} \int_{\tau_{0}}^{\theta} \widetilde{\Omega}_{m}(\tau) d \tau & =\omega\left(\tau_{0}\right)+\frac{1}{\theta-\tau_{0}} \int_{\tau_{0}}^{\theta}\left(f(\tau)+C \Delta\left|f^{\prime}(\tau)\right|\right) d \tau \\
& \geqq \omega\left(\tau_{0}\right)+\int_{\tau_{0}}^{\theta}\left(-\left|f^{\prime}(\tau)\right|+\frac{C \Delta}{\theta-\tau_{0}}\left|f^{\prime}(\tau)\right|\right) d \tau \geqq \omega\left(\tau_{0}\right)
\end{aligned}
$$

(to prove the last inequality we use that $\left.f\left(\tau_{0}\right)=f(\theta)=0\right)$. Since $\widetilde{\Omega}_{m} \geqq \omega_{m} \geqq \omega\left(\tau_{0}\right)$ in $\left[\theta, \tau_{*}\right]$ then $(4.7)$ follows.

Proposition 2. For $\tau_{2}>\tau_{1}+3 C \Delta$ we have

$$
\frac{1}{\tau_{2}-\tau_{1}} \int_{\tau_{1}}^{\tau_{2}} \widetilde{\Omega}_{m}(\tau) d \tau \geqq \frac{1}{3} C_{1}^{-1} R^{\mu} .
$$

The constants $C, C_{1}$ are the same as in Proposition 1. 
Proof. Let $a_{1} \geqq \tau_{1}$ be the first moment when the trajectory enters $\overline{\mathfrak{A}}$ and $b_{1} \geqq a_{1}$ be the moment of its first exit from $\overline{\mathfrak{A}}$. Let $a_{2}=\tau_{*}\left(b_{1}\right)>b_{1}$, where $\tau_{*}$ is as in the lemma (with $\tau_{0}=b_{1}$ ). Let $b_{2} \geqq a_{2}$ be the moment of the next exit from $\overline{\mathfrak{A}}$, etc.

We consider only the worst situation when $a_{1}>\tau_{1}$ and $b_{r}<\tau_{2}<a_{r+1}$ for some $r$. Since at each segment $\left[a_{j}, b_{j}\right]$ we have $\widetilde{\Omega}_{m} \geqq \omega_{m} \geqq C_{1}^{-1} R^{\mu}$ (see (4.5)), then averaging of $\widetilde{\Omega}_{m}$ along $\left[a_{j}, b_{j}\right]$ is $\geqq C_{1}^{-1} R^{\mu}$. Averaging of $\widetilde{\Omega}_{m}$ along any segment $\left[b_{j}, a_{j+1}\right]$ by the lemma also is $\geqq C_{1}^{-1} R^{\mu}$. Therefore averaging of $\widetilde{\Omega}_{m}$ along $\left[a_{1}, b_{r}\right]$ is $\geqq C_{1}^{-1} R^{\mu}$. follows.

By (4.4), $\left|a_{1}-\tau_{1}\right|+\left|\tau_{2}-b_{r}\right| \leqq 2 C \Delta$. So $\left|\tau_{2}-\tau_{1}\right| \leqq 3\left|b_{r}-a_{1}\right|$ and (4.8)

The number $\Delta$ contains the factor $\delta^{-v+1 / 2}$, where $v>1 / 2$ and the factor is large for small $\delta$. We can drop it, simultaneously changing the r.h.s. of (4.6), (4.8). Indeed, by Remark 1 we can replace $\mu$ and $v$ by $\widetilde{\mu} \leqq \mu, \tilde{v}=\frac{2 m+1}{m} \widetilde{\mu}$. Since $\mu \geqq 1 / 4$ (see $(2.2)$ ), then we can take $\mu=m /(4 m+2)<1 / 4$. Then $\stackrel{m}{\tilde{v}}=1 / 2$ and we get versions of the Propositions 1 and 2:

Proposition 1'. For $\tau_{2} \geqq \tau_{1}+C / \sqrt{E}$ we have

$$
\sup _{\tau_{1} \leqq \tau \leqq \tau_{2}} \omega_{m}(\tau) \geqq C^{-1} R^{m /(4 m+2)} .
$$

Proposition $2^{\prime}$. If $\tau_{2} \geqq \tau_{1}+3 C / \sqrt{E}$, then

$$
\frac{1}{\tau_{2}-\tau_{1}} \int_{\tau_{1}}^{\tau_{2}}\left(\omega_{m}+\frac{C}{\sqrt{E}}\left|\omega_{m}^{\prime}\right|\right) d \tau \geqq \frac{1}{3} C^{-1} R^{\frac{m}{4 m+2}} .
$$

We note that since $\mu(m)$ tends to $1 / 2$ as $m$ grows, then for large $m$ the exponents of $R$ in the r.h.s.'s of $\left(4.6^{\prime}\right),\left(4.8^{\prime}\right)$ are almost twice as small as in (4.6), (4.8).

If we treat (4.1) as a partial differential equation rather than a dynamical system in $Z$, then it is more natural to choose as a measure of space-time oscillations of a solution $u$ not $\widetilde{\Omega}_{m}$ but the function $\Omega_{m}$,

$$
\Omega_{m}(\tau)=\frac{\|u(\tau)\|+C^{\prime} \Delta\left\|u^{\prime}(\tau)\right\|}{|u(\tau)|_{\infty}}
$$

with $C^{\prime}$ as in (4.3). Observe that

$$
\Omega_{m}(\tau) \geqq \frac{\|u(\tau)\|+C^{\prime} \Delta\left|\frac{\partial}{\partial \tau}\|u(\tau)\|\right|}{|u(\tau)|_{\infty}} .
$$

We think that the quantity $\Omega_{m}$ is a natural measure for solution's oscillations and name the related statement "theorem":

Theorem 3. If $\tau_{2} \geqq \tau_{1}+3 C^{\prime} \Delta$, then

$$
\frac{1}{\tau_{2}-\tau_{1}} \int_{\tau_{1}}^{\tau_{2}} \Omega_{m}(\tau) d \tau \geqq \frac{1}{6} C_{1}^{-1} R^{\mu} ;
$$

if $\tau_{2} \geqq \tau_{1}+3 C / \sqrt{E}$, then

$$
\frac{1}{\tau_{2}-\tau_{1}} \int_{\tau_{1}}^{\tau_{2}} \frac{\|u(\tau)\|+C E^{-1 / 2}\left\|u^{\prime}(\tau)\right\|}{|u(\tau)|_{\infty}} d \tau \geqq \frac{1}{6} C_{2}^{-1} R^{\frac{m}{4 m+2}} .
$$


Proof of (4.10) essentially follows the proof of Proposition 2 . Let $b_{1} \geqq a_{1} \geqq \tau_{1}$ be the numbers from that proof and $b_{1}^{2}>b_{1}$ be the first moment of doubling the solution's norm. If $u\left(b_{1}^{2}\right) \notin \mathcal{U}$, let $b_{1}^{3}$ be the moment of the second doubling, etc. Finally, we shall find $b_{1}^{j} \in \mathfrak{A}, j \geqq 2$, and by (4.3),

$$
\left|b_{1}^{p}-b_{1}^{p-1}\right| \leqq C^{\prime} \Delta \quad \forall p \leqq j .
$$

Denote $a_{2}=b_{1}^{j}$, denote by $b_{2}$ the moment of the next exit from $\overline{\mathfrak{I}}$, construct $b_{2}, b_{2}^{2}, \ldots, a_{3}$ as above, etc.

Clearly, the average of $\Omega_{m}$ along each segment $\left[a_{r}, b_{r}\right]$ (or along $\left[a_{r}, \tau_{2}\right]$ if $\left.a_{r}<\tau_{2}<b_{r}\right)$ is $\geqq C^{-1} R^{\mu}$. To study a segment $\left[b_{r}^{p}, b_{r}^{p+1}\right]$ let us take any sequence $b_{r}, b_{r}^{2}, \ldots, b_{r}^{j}=a_{r+1}$ and denote for short

$$
x_{1}=b_{r}, \quad x_{2}=b_{r}^{2}, \ldots, x_{j}=b_{r}^{j}=a_{r+1} .
$$

As in the proof of the lemma,

$$
\omega\left(x_{1}\right) \leqq \omega\left(x_{2}\right) \leqq \cdots \leqq \omega\left(x_{j}\right) .
$$

Besides for each $\tau \in\left[x_{p}, x_{p+1}\right]$ we have $|u(\tau)|_{\infty} \leqq 2\left|u\left(x_{p}\right)\right|_{\infty}$. By this inequality and (4.9), (4.12) for each $p<j$ we have:

$$
\begin{aligned}
& \frac{1}{x_{p+1}-x_{p}} \int_{x_{p}}^{x_{p+1}} \Omega_{m}(\tau) d \tau \\
& \geqq \frac{1}{2\left(x_{p+1}-x_{p}\right)\left|u\left(x_{p}\right)\right|_{\infty}} \int_{x_{p}}^{x_{p+1}}\left(\|u(\tau)\|+C^{\prime} \Delta\left|\frac{\partial}{\partial \tau}\|u(\tau)\|\right|\right) d \tau \\
& \geqq \frac{\left\|u\left(x_{p}\right)\right\|}{2\left|u\left(x_{p}\right)\right|_{\infty}}+\frac{1}{2\left|u\left(x_{p}\right)\right|_{\infty}} \int_{x_{p}}^{x_{p+1}}\left(-\left|\frac{\partial}{\partial \tau}\|u\|\right|+\frac{C^{\prime} \Delta}{x_{p+1}-x_{p}}\left|\frac{\partial}{\partial \tau}\|u\|\right|\right) d \tau \\
& \geqq \frac{\left\|u\left(x_{p}\right)\right\|}{2\left|u\left(x_{p}\right)\right|_{\infty}}=\frac{1}{2} \omega\left(x_{p}\right),
\end{aligned}
$$

where we have used the elementary inequality $A^{-1} \int_{0}^{A} f(t) d t \geqq f(0)-\int_{0}^{A}\left|f^{\prime}(t)\right| d t$, valid for each $C^{1}$-smooth $f$.

Now (4.13) implies that

$$
\frac{1}{x_{p+1}-x_{p}} \int_{x_{p}}^{x_{p+1}} \Omega_{m}(\tau) d \tau \geqq \frac{1}{2} C_{1}^{-1} R^{\mu} .
$$

So we estimated the averages along all the segments with the possible exception of the segments $\left[\tau_{1}, a_{1}\right]$ (and $\left[b_{r}^{j}, \tau_{2}\right]$ if $\tau_{2} \in\left[b_{r}^{j}, b_{r}^{j+1}[\right.$ ). Since both the segments are shorter than $C^{\prime} \Delta$, the result follows.

The estimate (4.11) results from (4.10) in the same way as Proposition $2^{\prime}$ from Proposition 2.

\section{Some Generalizations}

5.1. Periodic Boundary Conditions. The Eq. (1.1) can be studied under periodic boundary conditions - i.e., in the whole space $H$ of the periodic functions 
$u(x), x \in T^{n}$ (see (1.4)). In this case for $c>0$ we should consider the subsets $\mathscr{L}_{c} \subset H$,

$$
\mathscr{L}_{c}=\left\{u(x) \in H|| u(x) \mid=c \forall x \in T^{n}\right\} .
$$

The sets $\mathscr{L}_{c}$ are smooth Lagrangian submanifolds of the phase-space $H$ which is given the symplectic structure defined in Part 1 . Next we define the "frame" $\mathscr{L}$ as the union of all $\mathscr{L}_{c}$,

$$
\mathscr{L}=\cup_{c} \mathscr{L}_{c}
$$

and define essential part $\mathfrak{U}_{p}$ of the phase-space $H$ as

$$
\mathfrak{A}_{p}=\left\{u(x) \mid \operatorname{dist}_{\infty}(u, \mathscr{L}) \leqq K \delta^{\mu}\|u\|^{1-2 \mu}\right\}
$$

Theorem 2 remains true for the periodic boundary value problem for Eq. (1.1) if we replace $\mathfrak{A}$ by $\mathfrak{A}_{p}$, cf. [8] where a weaker form of this result was obtained for nonlinear wave equations.

5.2. Polynomial Nonlinearities. Theorems 1 and 2 and propositions from Part 4 remain true (after correcting the constants $K, K_{1}$ and the exponents $\mu$ and $v$ ) if we replace the nonlinearity $|u|^{2} u$ by any $|u|^{2 p} u$ ( $p$ is a positive integer) or by a polynomial

$$
\left(a_{1}|u|^{2}+a_{2}|u|^{4}+\cdots+a_{p}|u|^{2 p}\right) u,
$$

where $a_{j} \geqq 0$ for all $j$ and $a_{p}>0$. The proofs go without any changes.

5.3. Focusing Equation. Consider Eq. (1.1) with $V=0$ and with the changed sign in front of the Laplacian:

$$
-i \dot{u}=\delta \Delta u+|u|^{2} u .
$$

If the phase-space $Z$ is a Sobolev space as in Examples 1 and 2 from Part 1, then solutions of (5.1) in $Z$ are well-defined locally in time (see e.g., [2]) but can blow up in finite time if $n>1$ (see [6]). So (5.1) defines in $Z$ a local flow. Still statements of Theorems 1 and 2 remain true with the natural refinement that the solutions of (5.1) exist until the correspondent times $t_{1}$ and $t^{\prime}, t^{\prime \prime}$. The only difference comes during proving the second statement of Theorem 2 since in the focusing case (5.1) we cannot use the Hamiltonian $\mathscr{H}$ to majorize the $L^{4}$-norm of the solution. Instead we can use in the estimate (3.5) the $L^{2}$-norm (which still is an integral of motion) under the additional restriction:

$$
m \geqq 4 \text { if } n=3 \text {. }
$$

5.4. Local Flow. Suppose that a phase space $Z \subset C^{m}$ for the problem (1.1), (1.2) is chosen in such a way that solutions $u(t) \in Z$ are defined only locally in time (or we cannot prove that they are defined globally, as in the case when $n=2,3, V(x) \neq 0$ and $Z$ is the Sobolev space as in Example 2). Then - as in the previous item - the statements of Theorems 1, 2 remain true with the same obvious refinement that the solutions do exist till the times $t_{1}, t^{\prime}, t^{\prime \prime}$.

Acknowledgements. This paper was done at the Institute for Advanced Study (Princeton). I sincerely thank I.A.S. for their hospitality and Tom Spencer for useful discussions. 


\section{References}

1. Adams, R.S.: Sobolev spaces. New York: Academic Press, 1975

2. Bourgain, J.: Fourier transform restriction phenomena for certain lattice subsets and applications to nonlinear evolution equations. Geom. and Funct. Anal. 3, 107-156 and 209-262 (1993)

3. Bourgain, J.: Quasi-periodic solutions of Hamiltonian perturbations of 2D linear Schrödinger equations. Preprint (1994)

4. Il'yashenko, Ju. S.: Global analysis of the phase-portrait of the Kuramoto-Sivashinsky equation. J. Dynamics Diff. Eq. 4, 585-615 (1992)

5. Jin, S., Levermore, C.D., McLaughlin D.W.: The semiclassical limit of the defocusing NLS hierarchy. Preprint (1995)

6. Kavian, O.: A remark on the blowing-up of solutions of the Cauchy problem for nonlinear Schrödinger equation. Trans. Am. Math. Soc. 299, 193-203 (1987)

7. Kuksin, S.B.: Nearly integrable infinite-dimensional Hamiltonian systems. Lecture Notes Math. 1556, Berlin-Heidelberg-New York: Springer, 1993

8. Kuksin, S.B.: On squeezing and flow of energy for nonlinear wave equations. Geom. and Funct. Anal. 5:4 (1995)

9. Kuksin, S.B.: Infinite-dimensional symplectic capacities and a squeezing theorem for Hamiltonian PDE's. Commun. Math. Phys. 167, 531-552 (1995)

10. Monin, A.S., Yaglom, A.M.: Statistical fluid mechanics: Mechanics of turbulence, Vol. 1. Moscow: Nauka Press, 1965; English trans. Cambridge-London: The MIT Press, 1971

11. Pomeau, Y.: Asymptotic time behavior of nonlinear classical field equations. Nonlinearity $\mathbf{5}$, 707-720 (1992)

Communicated by A. Kupiainen 